\title{
L’immunothérapie, une révolution en oncologie
}

$>$ L'utilisation des inhibiteurs de points de contrôle immunitaire a révolutionné la prise en charge et le pronostic de nombreux patients atteints de cancer. L'arrivée de ces nouveaux traitements s'est accompagnée de la découverte de nouveaux effets indésirables nécessitant des prises en charge spécifiques. De plus, l'évolution tumorale et sa surveillance sous immunothérapie est différente de celle sous traitements classiques, et ont nécessité une adaptation des critères radiologiques et du suivi des lésions tumorales. De nombreuses autres cibles thérapeutiques existent, et pourraient potentiellement être associées aux inhibiteurs des points de contrôle immunitaires. II existe donc encore de nombreux défis à relever afin de mieux comprendre et d'optimiser l'utilisation de ces nouvelles molécules. <

\section{Toxicités}

Les effets indésirables des inhibiteurs de points de contrôle immunitaire sont différents de ceux des chimiothérapies classiques. La levée d'inhibition du système immunitaire peut conduire à la survenue d'effets indésirables auto-immuns selon des mécanismes restant mal connus.

Ils apparaissent le plus souvent de façon retardée, habituellement dans les 12 premières semaines de traitement, mais peuvent survenir jusqu'à 6 mois après l'arrêt du traitement. Ils peuvent atteindre tous les organes, avec un tropisme particulier pour les glandes endocriniennes (thyroïdite, hypophysite), le tube digestif (colite), le foie (hépatite), les poumons (pneumopathie interstitielle) et la peau (prurit, vitiligo, rash).

Un des effets indésirables le plus fréquemment rapporté est l'asthénie (autour de $15 \%$ pour les patients traités par anticorps anti-PD-1/PDL-1 $[1,2]$ et jusqu'à près de $50 \%$ pour ceux traités par les anticorps antiCTLA-4 [3]), ainsi que l'hyperthermie (environ $10 \%$ des patients traités par anti-PD-1).

\section{Spécificités}

de l'immunothérapie pour le clinicien

\section{Manon Dubois ${ }^{1 *}$, Camille Ardin ${ }^{2 *}$, Fanny André ${ }^{1}$, Arnaud Scherpereel ${ }^{2-4}$, Laurent Mortier ${ }^{1,3,4}$}

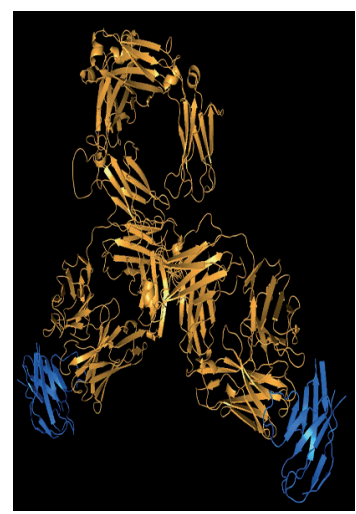

Fréquentes mais rarement graves, les atteintes rapportées sont digestives ( $10 \%$ des patients sous anti-PD-1/-PD-Ll, et jusqu'à $40 \%$ sous anti-CTLA-4), avec notamment des diarrhées et des nausées. Les atteintes cutanées rapportées par environ 40-45\% des patients sous anti-CTLA-4, et entre 10 et $20 \%$ des patients sous anti-PD-1/-PD-L1 [4], se manifestent principalement par un prurit ou un érythème. Un vitiligo peut également survenir, associé à une meilleure réponse au traitement [5]. Les toxicités endocrinologiques sont plus fréquentes sous anti-CTLA-4 (près de $10 \%$ des patients), à type d'hypophysites (9-18\%) ou de thyroïdites (2-10\%). On observe également, de façon plus rare, la survenue de diabètes pouvant nécessiter une insulinothérapie substitutive. Les atteintes pulmonaires surviennent chez près de $5 \%$ des patients traités par anticorps anti-CTLA-4 ou anti-PD-1/-PD-L1. Le profil de toxicité diffère selon l'agent utilisé : les colites auto-immunes et les hypophysites semblent plus fréquentes chez les patients exposés aux anticorps anti-CTLA-4, alors que les atteintes pulmonaires autoimmunes et les dysthyroïdies serait plus fréquentes chez les patients recevant des anticorps anti-PD-1 [6]. Les données récentes suggèrent aussi que les effets indésirables auto-immuns sont plus précoces, plus fréquents et plus sévères dans les traitements anti-CTLA-4 que les traitements anti-PD-1/PD-Ll. Enfin, des effets secondaires graves mais rares ont été décrits : des atteintes myocardiques pouvant se présenter sous la forme de myocardites, ainsi que des effets secondaires neurologiques, avec des atteintes centrales (encéphalites) ou périphériques (tableaux de Guillain Barré ou apparentés). 
Selon la sévérité de l'atteinte, la prise en charge ira de la suspension temporaire du traitement à son arrêt définitif, associé à l'introduction d'une corticothérapie, voire d'un traitement immunosuppresseur [7].

\section{Phénomène d'hyperprogression}

Certains patients présentent une progression anormalement rapide de leur maladie sous inhibiteurs des points de contrôle immunitaires, une «hyperprogression» dont le pronostic est très péjoratif. Ce phénomène n'est pas rattaché à un type précis de néoplasie, mais il se produirait chez $8 \%$ à $21 \%$ des patients atteints d'un cancer pulmonaire, chez $10 \%$ des patients traités pour un mélanome et jusqu'à $30 \%$ des patients atteints de cancer oto-rhino-laryngologique. Les mécanismes à l'origine de ce phénomène restent imparfaitement connus, et son existence même reste discutée : I'hyperprogression se produirait également chez des patients traités par chimiothérapie ou non traités, et son mécanisme immunologique n'est, à ce jour, pas prouvé [8].

Finalement, les effets indésirables des inhibiteurs de points de contrôle restent très majoritairement d'une sévérité modérée (environ $10 \%$ de patients déclarant un effet indésirable de grade 3 à $5[1,2]$ ), et la qualité de vie des patients ainsi traités est meilleure que celle des patients exposés à la chimiothérapie [9].

\section{Marqueurs prédictifs de réponse à l'immunothérapie}

Si les inhibiteurs de points de contrôle peuvent être considérés comme une révolution thérapeutique dans la prise en charge de certains cancers avancés, ils ne sont pas pour autant efficaces chez tous les patients. Nous avons vu que, s'ils sont d'une façon générale bien tolérés, ils ne sont pas non plus dénués d'effets indésirables pouvant être graves. Il s'agit donc de tenter de sélectionner les meilleurs patients candidats à cette immunothérapie.

Le taux d'expression tumorale de PD-Ll semble être un marqueur prédictif logique de réponse au traitement par anticorps anti-PD-1/ PD-Ll. Cependant, comme le montre l'essai Keynote-024, même dans une population très sélectionnée de patients exprimant fortement PD-Ll (supérieur à $50 \%$ ), le taux de réponse au traitement est inférieur à $50 \%$. Dans le mélanome, le taux d'expression de PD-Ll n'est pas corrélé au taux de réponse au traitement [10]. Plusieurs études tentent d'identifier les relations de la réponse avec la charge mutationnelle (TMB) [11], la signature génique de l'activation lymphocytaire «T eff » [12], le statut épigénétique de méthylation du gène FOXPI sur échantillons tumoraux [13], ou encore l'évaluation de la signature radiomique ${ }^{1}$ de la lésion [14].

\section{Évaluation de la réponse au traitement}

Les inhibiteurs de points de contrôle immunitaire, contrairement à la chimiothérapie, n'ont pas d'action cytotoxique directe. Lors de la phase initiale du traitement, il est possible d'observer une augmentation de

${ }^{1}$ Évaluation par scanner du caractère infiltré ou non de la tumeur par des lymphocytes. volume des lésions tumorales, en lien avec l'infiltration de la tumeur par les lymphocytes T, suivie d'une régression ou d'une stabilisation des lésions à l'évaluation suivante. Cet aspect de bon pronostic, appelé «pseudoprogression » peut être observé dans $5 \%$ des cas. De nouveaux critères radiologiques (irRC, iRecist) sont en cours de validation et tentent de répondre à ces évolutions atypiques [15]. Cette «pseudo-progression» reste actuellement encore difficile à différencier d'une progression vraie en routine. L'association d'un marqueur biologique, comme le dosage de la quantité d'ADN tumoral circulant, pourrait être une aide dans l'appréciation de ces pseudo-progressions notamment dans le mélanome métastatique [16].

\section{Peut-on arrêter l'immunothérapie?}

Il n'existe pas, à ce jour, de recommandation sur la durée optimale de traitement par inhibiteurs des points de contrôle immunitaires. Les données disponibles montrent que les patients répondeurs au traitement maintiennent cette réponse de façon prolongée. L'essai Keynote-006 [17] prévoyait, pour les patients atteints d'un mélanome métastatique inclus dans le bras pembrolizumab, un traitement durant 2 ans. Dix-huit pour cent des patients ont effectivement pu poursuivre le traitement durant 2 ans. Vingt mois après l'arrêt du traitement chez ces patients, $86 \%$ n'avait pas présenté de rechute. Ce taux de contrôle de la maladie était encore meilleur chez les patients en réponse complète sous immunothérapie ( $95 \%$ ) ou chez les patients en réponse partielle (91\%). Parmi les patients en progression (18\%), 8 ont bénéficié d'un nouveau traitement par pembrolizumab : 4 ont répondu à nouveau à ce traitement, 3 ont stabilisé leur maladie et 1 seul patient a présenté une progression.

\section{Conclusion}

Les résultats des inhibiteurs de points de contrôle immunitaires sont prometteurs et la gestion de leur effets indésirables de mieux en mieux codifiée. De nombreuses questions restent en suspens, telles que la recherche de marqueurs prédictifs de réponse au traitement, la personnalisation du traitement en fonction du profil tumoral ou les possibilités de combinaisons et d'arrêts de traitement.

La place des inhibiteurs des points de contrôle immunitaire dans les protocoles de traitement fait actuellement l'objet de nombreux essais thérapeutiques et devrait notablement évoluer dans les prochaines années. $\diamond$ 


\section{SUMMARY}

The revolution of immuno-oncology therapy: specificities for the physicians

The use of immune checkpoint inhibitors has revolutionized the treatment and prognosis of many cancer patients. Associated with the raise of these new treatments, new side effects have been observed, requiring specific management. In addition, the tumor evolution and its monitoring under immunotherapy differ from conventional treatments, and require an adaptation of the radiological criteria for tumor lesions monitoring. Many other therapeutic targets exist and could potentially be associated with immune checkpoint inhibitors. Many challenges still need to be overcome in order to better understand and optimize the use of these new molecules. $\diamond$

\section{LIENS D'INTÉRÊT}

Les auteurs déclarent n'avoir aucun lien d'intérêt concernant les données publiées dans cet article.

\section{RÉFÉRENCES}

1. Reck M, Rodríguez-Abreu D, Robinson AG, et al. Pembrolizumab versus chemotherapy for PD-LIpositive non-small-cell lung cancer. N Engl J Med 2016 ; 375 : 1823-33.

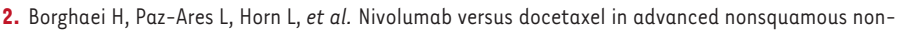
small-cell lung cancer. N Engl J Med 2015 ; 373 : 1627-39.

3. Eggermont AMM, Chiarion-Sileni V, Grob JJ, et al. Adjuvant ipilimumab versus placebo after complete resection of high-risk stage III melanoma (EORTC 18071): a randomised, double-blind, phase 3 trial. Lancet Oncol $2015 ; 16: 522-30$.

4. Boutros C, Tarhini A, Routier $\varepsilon$, et al. Safety profiles of anti-CTLA-4 and anti-PD-1 antibodies alone and in combination. Nat Rev Clin Oncol 2016 ; 13 : 473-86.

5. Hua C, Boussemart L, Mateus C, et al. Association of vitiligo with tumor response in patients with metastatic melanoma treated with pembrolizumab. JAMA Dermatol 2016 ; 152 : 45-51.

6. Postow MA, Sidlow R, Hellmann MD. Immune-related adverse events associated with immune checkpoint blockade. $N$ Engl J Med 2018 ; 378 : 158-68.

7. Johnson DB, Chandra S, Sosman JA. Immune checkpoint inhibitor toxicity in 2018. JAMA $2018 ; 320$ : 1702-3.
8. Chouaid C, Rousseau-Bussac G, Monnet I. Qualité de vie des patients atteints de cancer du poumon traités par immunothérapie. Rev Mal Respir Actual $2018 ; 10: 487-90$.

9. Wasielewski $\varepsilon$, Cortot $A B$. Tumour assessment criteria for immune checkpoint inhibitors. Rev Mal Respir 2018 ; 35 : 828-45.

10. Lee JH, Long GV, Menzies AM, et al. Association between circulating tumor DNA and pseudoprogression in patients with metastatic melanoma treated with anti-programmed cell death 1 antibodies. JAMA Oncol $2018 ; 4$ : 717 21.

11. Champiat S, Ferrara R, Massard C, et al. Hyperprogressive disease: recognizing a novel pattern to improve patient management. Nat Rev Clin Oncol 2018; $15: 748-62$.

12. Adam J, Tomasic G, Robert C. Tissue biomarkers of response to anti-PD-1 immunotherapies in melanoma. Ann Pathol $2017 ; 37: 55-60$.

13. Hellmann MD, Ciuleanu T- $\varepsilon$, Pluzanski $A$, et al. Nivolumab plus Ipilimumab in lung cancer with a high tumor mutational burden. N Engl J Med 2018 ; 378 : 2093-104.

14. Socinski MA, Jotte RM, Cappuzzo F, et al. Atezolizumab for first-line treatment of metastatic nonsquamous NSCLC. N Engl J Med 2018 ; 378 : 2288-301.

15. Duruisseaux M, Martínez-Cardús A, Calleja-Cervantes ME, et al. Epigenetic prediction of response to anti-PD- 1 treatment in non-small-cell lung cancer: a multicentre, retrospective analysis. Lancet Respir Med 2018 ; 6 : 771-81.

16. Sun $R$, Limkin $\varepsilon J$, Vakalopoulou $M$, et al. A radiomics approach to assess tumour-infiltrating CD8 cells and response to anti-PD-1 or anti-PD-LI immunotherapy: an imaging biomarker, retrospective multicohort study. Lancet Oncol 2018; 19 : 1180-91.

17. Schachter J, Ribas A, Long GV, et al. Pembrolizumab versus ipilimumab for advanced melanoma: final overall survival results of a multicentre, randomised, open-label phase 3 study (Keynote-006). Lancet 2017 ; 390 : 1853-62.
TIRÉS À PART

L. Mortier

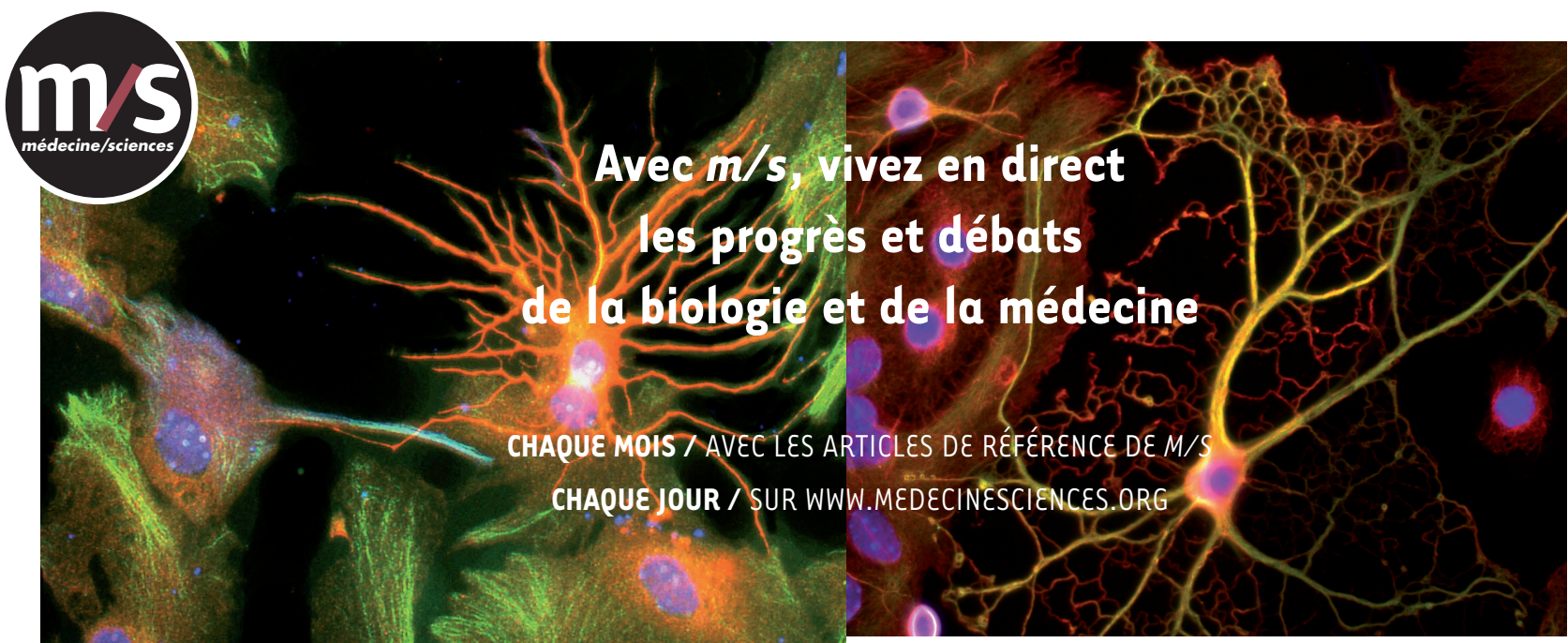

Abonnez-vous sur

www.medecinesciences.org 